\title{
BMJ Open Australian Suicide Prevention using Health-Linked Data (ASHLi): Protocol for a population-based case series study
}

\author{
Kate M Chitty, ${ }^{1}$ Jennifer L Schumann, ${ }^{2}$ Andrea Schaffer (1) , ${ }^{3}$ Rose Cairns (i) , ${ }^{4,5}$ \\ Nicole J Gonzaga (D) , ${ }^{1}$ Jacques E Raubenheimer, ${ }^{1}$ Gregory Carter, ${ }^{6}$ Andrew Page, ${ }^{7}$ \\ Sallie-Anne Pearson, ${ }^{3}$ Nicholas A Buckley ${ }^{1}$
}

To cite: Chitty KM, Schumann JL, Schaffer A, et al. Australian Suicide Prevention using HealthLinked Data (ASHLi): Protocol for a population-based case series study. BMJ Open 2020;10:e038181. doi:10.1136/ bmjopen-2020-038181

- Prepublication history for this paper is available online. To view these files, please visit the journal online (http://dx.doi. org/10.1136/bmjopen-2020038181).

Received 10 March 2020 Revised 09 April 2020 Accepted 14 April 2020
Check for updates

(C) Author(s) (or their employer(s)) 2020. Re-use permitted under CC BY-NC. No commercial re-use. See rights and permissions. Published by BMJ.

For numbered affiliations see end of article.

Correspondence to Dr Kate M Chitty; kate.chitty@sydney.edu.au

\section{ABSTRACT}

Introduction In Australia, suicide is the leading cause of death for people aged 15-44 years. Health professionals deliver most of our key suicide prevention strategies via health services, but other efficacious population-level strategies include means restriction and public awareness campaigns. Currently, we have no population-level data allowing us to determine which individuals, in what parts of Australia, are likely to use our most promising interventions delivered by health services. The aims of this study are to describe: (1) health service utilisation rates in the year prior to death by suicide, and how this varies by individual case characteristics; (2) prescribed medicines use in the year prior to death by suicide, medicines used in suicide by poisoning and how this varies by individual case characteristics.

Methods and analysis This is a population-based case series study of all suicide cases in Australia identified through the National Coronial Information System (NCIS) from 2013 to 2019. Cases will be linked to administrative claims data detailing health service use and medicines dispensed in the year before death. We will also obtain findings from the coronial enquiry, including toxicology. Descriptive statistics will be produced to characterise health service and prescribed medicine use and how utilisation varies by age, sex, method of death and socioeconomic status. We will explore the geographical variability of health service and medicine use, highlighting regions in Australia associated with more limited access.

Ethics and dissemination This project involves the use of sensitive and confidential data. Data will be linked using a third-party privacy-preserving protocol meaning that investigators will not have access to identifiable information once the data have been linked. Statistical analyses will be carried out in a secure environment. This study has been approved by the following ethics committees: (1) the Justice Department Human Research Ethics Committee (REF: CF/17/23250), (2) the Western Australian Coroners Court (REF: EC 14/18 M0400), (3) the Australian Institute of Health and Welfare (REF: E02017/4/366) and (4) NSW Population \& Health Services Research Ethics Committee (REF: 2017/HRE1204). Findings will be published in peer-reviewed journals, presented at conferences and communicated to regulatory authorities, clinicians and policy-makers.
Strengths and limitations of this study

- This is a population-based case series study of all suicide cases in Australia from 2013 to 2019.

- Coronial data will be linked to individual dispensing records for prescribed medicines to create a comprehensive view of medicine access at the time of death.

- By measuring the geographical variability of health service use across the cohort, we will highlight regions in Australia with reduced access to these important avenues for suicide prevention.

- All cases in the study have died from suicide, therefore, the risk of suicide cannot be established.

\section{INTRODUCTION}

\section{Background}

Suicide prevention is a worldwide public health priority. ${ }^{1}$ In Australia, suicide is the leading cause of years of potential life lost, the leading cause of death for people aged between 15 and 44 years, and suicide rates have remained largely unchanged over the past decade. $^{2}$

The most effective suicide prevention strategies delivered by health services in Australia include training general practitioners (GPs) to identify and support people in distress, similar training for other health personnel who are likely to encounter individuals at risk ('gatekeepers') and implementation of psychosocial treatments. ${ }^{3}$ However, we do not have robust estimates of the proportion of people who will visit a GP, have contact with gatekeepers or receive psychosocial treatments before they die from suicide. In order to best estimate the scope of these promising interventions and, accordingly, reinforce or redirect our efforts, we need to know what health services were, and were not, utilised by people in Australia in the period prior to suicide. 
Psychotropic medicines have complex modifying effects on the risk of suicide, which may change substantially with age, indication, agent and duration. ${ }^{4}$ These medicines are also some of the most common substances used in suicide by poisoning. ${ }^{5}$ Currently, however, we do not know what medicines have been prescribed to people who die of suicide, and what proportion use their prescribed medicines for self-poisoning. This is the key information for prescribers and could influence future means restriction activities.

The Australian Suicide Prevention using Health-Linked Data (ASHLi) project is a population-based case series study. The overarching aims are to describe and characterise the following for all suicides in Australia between 2013 and 2019:

(1) health service utilisation rates (both general and mental health) in the year prior to death by suicide, and how this varies by individual case characteristics; and (2) prescribed medicines use in the year prior to death by suicide, medicines used in suicide by poisoning and how this varies by individual case characteristics.

\section{Summary of the existing literature}

Healthcare utilisation prior to suicide using administrative claims data

The most recent review of healthcare utilisation prior to suicide $^{6}$ incorporated 13 population-based studies using administrative claims and mortality data from six jurisdictions (USA, Denmark, Taiwan, Republic of Korea, Canada and Sweden). The study found that contact with primary healthcare professionals in the month before suicide ranged between 50\% (USA between 2000 and 2010) and 73\% (Taiwan, 2006 and Canada, 1992-2000). In the 12 months preceding suicide, contact with a primary health professional ranged between $80 \%$ (Taiwan, 2001-2004) and 92\% (Canada, 1998-2011). The ranges were considerably lower when the focus was on mental health contacts alone: from 7\% (Sweden, 1991-2003) to 30\% (Canada, 1998-2011) in the month before suicide. In the 12 months prior to death, mental health contact ranged from $21 \%$ (UK, 1996-2005) to 25\% (Sweden, 1991-2003). Therefore, estimates of healthcare contact prior to suicide are highly variable, which may be partly due to differences in healthcare systems, study methodology and data quality. Importantly, there are pronounced within-country differences in healthcare contact based on characteristics such as sex, age ${ }^{6}$ and race/ethnicity. ${ }^{78}$

Medicine use prior to suicide using administrative claims data and toxicology findings at the time of death

Most analyses of medicine-linked data have been population-based cohort studies focused on examining suicide risk among all users of individual drug classes within a population. We identified only one retrospective study linking coroner records with person-level dispensing data. ${ }^{9}$ This Northern Ireland study investigated the odds of suicide associated with prescription of mental health and pain medicines, compared with living controls from the general population. The use of both mental health and pain medicines was associated with higher odds of suicide. ${ }^{10} 11$ This study also uncovered that recent prescription of these medicines (ie, in the 3 months preceding death) was associated with the highest risk of suicide, showing that pharmaceutical claims may have the ability to identify the emergence of mental health symptoms in the lead up to suicide.

Other information about prior medicine use in people who have died from suicide comes from toxicology studies that investigate medicines detected at autopsy. A study investigating the relative toxicity of a number of antidepressant medicines used in overdose reported wide differences in toxicity between classes and recommended caution in prescribing particular medicines to highrisk patients. ${ }^{12}$ A Canadian study reported that opioids, sedatives, tricyclic antidepressants and over-the-counter medicines were the most frequent substances detected in overdose from coroner's toxicology reports, again with authors advising caution in prescribing and careful follow-up. ${ }^{5}$

Autopsy studies to date have yielded important information for means restriction and clinical practice, yet interpreting medicine utilisation in autopsy-based analyses is subject to numerous limitations-it is unknown: (1) what medicines detected at autopsy were prescribed to the individual; or (2) whether medicines not detected are because the individual was not prescribed the medicine; prescribed the medicine but not adherent at the time of death; the samples collected were insufficient for toxicological analysis; or analyses conducted did not have the range or sensitivity to detect all therapeutic drug concentrations.

Healthcare and medicine use in the general Australian population In Australia, a wide range of health services are subsidised through the Medicare Benefits Scheme (MBS). Under the MBS, all Australian citizens and residents receive subsidised healthcare for out-of-hospital medical services and are guaranteed free treatment in public hospitals. Using the MBS, the Australian Institute of Health and Welfare (AIHW) reported that $88 \%$ of the Australian population visited a GP in 2017-2018, but that the rate of use varied considerably depending on where people lived. ${ }^{13}$ Data show that people living in remote areas have poorer access to, and the use of, health services compared with people living in major cities. ${ }^{14}$ In terms of mental health treatment, about $10 \%$ of the population received MBSsubsidised services in 2017-2018; this rate was higher in women $(12.2 \%)$ than men $(8.3 \%)$ and people aged $35-44$ years accessed mental health services the most $(13.8 \%$ of people in this age group). ${ }^{15}$

The Pharmaceutical Benefits Scheme (PBS) was established to subsidise a wide range of prescription medicines for all Australian citizens and residents. Using the PBS data collection, the AIHW determined that $16.8 \%$ of the Australian population received prescriptions for mental health related medicines in 2017-2018. Women had a 
Table 1 Number of suicide cases meeting study inclusion criteria per year, as identified by the NCIS access liaison officer

\begin{tabular}{lccl}
\hline & $\begin{array}{l}\text { Cases to be } \\
\text { linked (n)- } \\
\text { Yustralia }\end{array}$ & $\begin{array}{l}\text { Cases to } \\
\text { be linked } \\
\text { (n)-NSW }\end{array}$ & $\begin{array}{l}\text { Percentage } \\
\text { of all NCIS } \\
\text { cases closed } \\
\text { by coroner* }\end{array}$ \\
\hline $2013 \dagger$ & 1272 & 317 & 96.5 \\
\hline 2014 & 2760 & 753 & 95.0 \\
\hline 2015 & 2893 & 760 & 93.0 \\
\hline 2016 & 2693 & 692 & 92.9 \\
\hline 2017 & 2823 & 829 & 75.6 \\
\hline 2018 & 1813 & 585 & 50.8 \\
\hline 2019 & 285 & 128 & 19.9 \\
\hline Total & 14639 & 4064 & \\
\hline
\end{tabular}

${ }^{*}$ At the time of data extraction.

†Only cases after 1 July 2013 were used.

NCIS, National Coronial Information System; NSW, New South Wales.

higher proportion of mental health related prescriptions compared with men (20\% vs $13.6 \%$ ). Antidepressants were the most common mental health medicine prescribed, followed by anxiolytics, sedatives and antipsychotics. ${ }^{15}$

\section{METHODS AND ANALYSIS \\ Design}

ASHLi is a population-based case series study, which will detail the health service and medicine history of all suicides identified through the National Coronial Information System (NCIS) that occurred in Australian residents on/after 1 July 2013. All suicides will be linked to health service and medicine use that occurred in the year prior to death.

\section{Case definition}

The cases in our study are all deaths recorded by the NCIS as intentional or undetermined intent that occurred on/ after 1 July 2013 until the 10 October 2019 (date of data extraction) across all Australian jurisdictions according to the following inclusion criteria:

- Case closed by the coroner at the time of data extraction.

- Manner of death determined by the coroner to be intentional self-harm or undetermined intent.

The number of cases for each year are presented in table 1 .

Cases will also be confirmed using information from the National Death Index (NDI). Any suicide cases from the NCIS that are not coded as suicide in the NDI will still be treated as a case; however, the level of agreement between the two data sources will be reported. Only cases that are successfully linked to their PBS/MBS (for all cases) and New South Wales (NSW) Health records (for NSW cases) will be included in further analyses.
Data sources

Table 2 lists all the datasets that will be linked in ASHLi.

\section{Coronial data}

The National Coronial Information System (NCIS) captures Australia's coronial information for all deaths notified to a coroner since 2000 , it is managed by the Victorian Department of Justice and Community Safety. All deaths that are intentional, accidental or of undetermined intent are reviewed by a coroner and hence suicides are well captured. The NCIS carries detailed information, including demographics, autopsy and toxicology findings and details surrounding death (including method of death). Cases for which suicide is not a clear cause of death can be supported with information from the National Death Index (NDI), a national dataset of all causes of death held by the AIHW.

\section{Australia-wide administrative data}

Australia maintains a publicly funded, universal healthcare system entitling all citizens and permanent residents to subsidised medicines through the PBS. The PBS reimburses community pharmacies and private hospitals for PBS-listed medicines. ${ }^{16}$ The PBS data capture approximately $93 \%$ of prescription medicine use in Australia. ${ }^{17}$ The PBS does not subsidise medicines sold over the counter, complementary and alternative medicines, private prescriptions, medicines dispensed within public hospitals to inpatients or opioid maintenance treatments (methadone and buprenorphine) and, therefore, these medicines do not appear in the PBS collection. The MBS is Australia's government-funded healthcare subsidies scheme. The scheme subsidises a wide range of both public and private medical services including consultation fees for doctors and specialists, clinical psychology, diagnostic tests and examinations and most surgical and therapeutic procedures.

\section{NSW administrative data}

The NSW Emergency Department Data Collection (EDDC) collects data on presentations to emergency departments of public hospitals in NSW. The NSW Admitted Patient Data Collection (APDC) records all inpatient separations (discharges, transfers and deaths) from all public, private, psychiatric and repatriation hospitals in NSW, as well as public multipurpose services, private day procedure centres and public nursing homes. The NSW Mental Health Ambulatory Data Collection (MHAMB) includes information in relation to mental health day programmes, psychiatric outpatients and outreach services (eg, home visits). It also includes information regarding care provided by hospital-based consultationliaison services to admitted patients in non-psychiatric and hospital emergency settings and care provided by community workers and clients in staffed community residential settings, mental health promotion and prevention services. 
Table 2 Data sources to be linked in ASHLi

\begin{tabular}{|c|c|c|c|c|}
\hline Data set & Data description & Data provider & $\begin{array}{l}\text { Geographical } \\
\text { coverage }\end{array}$ & $\begin{array}{l}\text { Coverage in } \\
\text { years }\end{array}$ \\
\hline $\begin{array}{l}\text { Coronial cases } \\
\text { and information }\end{array}$ & $\begin{array}{l}\text { The National Coronial Information System (NCIS) } \\
\text { is a national repository containing data on deaths } \\
\text { reported to a coroner in Australia and New Zealand. }\end{array}$ & NCIS & Australia-wide & $\begin{array}{l}1 \text { July 2013-10 } \\
\text { October } 2019\end{array}$ \\
\hline $\begin{array}{l}\text { Medicines } \\
\text { dispensing } \\
\text { claims }\end{array}$ & $\begin{array}{l}\text { Australia's Pharmaceutical Benefits Scheme (PBS) is } \\
\text { a national drug subsidy programme for the approved } \\
\text { prescription medication and captures the dispensing } \\
\text { of all PBS-approved medicines in Australia. } \\
\text { Data will be extracted for each individual during the } \\
\text { exposure period. }\end{array}$ & AlHW & Australia-wide & $\begin{array}{l}1 \text { July } 2012- \\
\text { latest available } \\
\text { before death }\end{array}$ \\
\hline $\begin{array}{l}\text { Health service } \\
\text { records }\end{array}$ & $\begin{array}{l}\text { The Medicare Benefits Scheme (MBS) is a listing of } \\
\text { the Medicare services subsidised by the Australian } \\
\text { government including any healthcare contacts, and } \\
\text { any items used that are listed under the Mental } \\
\text { Health Plan. }\end{array}$ & AlHW & Australia-wide & $\begin{array}{l}1 \text { July } 2012- \\
\text { latest available } \\
\text { before death }\end{array}$ \\
\hline $\begin{array}{l}\text { Admitted patient } \\
\text { data }\end{array}$ & $\begin{array}{l}\text { The Admitted Patient Data Collection (APDC) } \\
\text { contains records of all inpatient separations } \\
\text { (discharges, transfers and deaths) from all public, } \\
\text { private, psychiatric and repatriation hospitals in } \\
\text { NSW. }\end{array}$ & $\begin{array}{l}\text { NSW Health } \\
\text { APDC }\end{array}$ & NSW & $\begin{array}{l}1 \text { July } 2012- \\
\text { latest available }\end{array}$ \\
\hline $\begin{array}{l}\text { Ambulatory } \\
\text { mental health } \\
\text { data }\end{array}$ & $\begin{array}{l}\text { The Mental Health Ambulatory (MH-AMB) Data } \\
\text { Collection is dedicated to the assessment, } \\
\text { treatment, rehabilitation or care of non-admitted } \\
\text { patients. It may include mental health day } \\
\text { programmes, psychiatric outpatients and outreach } \\
\text { services (eg, home visits). The data record 'contacts' } \\
\text { (as opposed to 'episodes of care') by clinicians to a } \\
\text { patient. }\end{array}$ & $\begin{array}{l}\text { NSW Health } \\
\text { MH-AMB data } \\
\text { collection }\end{array}$ & NSW & $\begin{array}{l}1 \text { July } 2012- \\
\text { latest available }\end{array}$ \\
\hline
\end{tabular}

AlHW, Australian Institute of Health and Welfare; APDC, Admitted Patient Data Collection

; ASHLi, Australian Suicide Prevention using Health-Linked Data ; EDDC, Emergency Department Data Collection; MH-AMB, Mental Health Ambulatory; NCIS, National Coronial Information System

; NDI, National Death Index; NSW, New South Wales; PBS, Pharmaceutical Benefits Scheme.

\section{Measurements}

Data from coroner reports

The information from the NCIS that will be extracted for each suicide is listed in table 3. Data extraction from the toxicology, police and autopsy reports will be completed by research assistants and students. Study investigators ( $\mathrm{KC}, \mathrm{RC}$ and JS) will provide training in the data extraction processes and will be available to address any questions and reach consensus regarding coding for more complex cases.

\section{Data from the PBS and MBS}

The data from the PBS that will be linked to each case include the date the medicine was prescribed and date the medicine was dispensed, medicine name, form, strength, number of prescriptions dispensed, and specialty of the prescriber. The data from the MBS that will be linked to each case include the date and type of health service accessed.

\section{Data from the APDC, EDDC and MH-AMB}

Data from the APDC and EDDC that will be linked to each case include dates of episodes of care, diagnosis codes associated with an episode of care, and mode of arrival and separation. Additionally, the triage category assigned to ED visits will be linked. Data from the MH-AMB that will be linked to each case include dates associated with 
Table 3 Information collected from the NCIS for each included suicide case

\begin{tabular}{|c|c|}
\hline Variable(s) & Description \\
\hline $\begin{array}{l}\text { Deidentified } \\
\text { demographic } \\
\text { variables }\end{array}$ & $\begin{array}{l}\text { Year of death, age at the time of death, sex, marital status, employment status, country of birth, years in } \\
\text { the country }\end{array}$ \\
\hline $\begin{array}{l}\text { ICD-10 - cause of } \\
\text { death }\end{array}$ & As determined during the Australian Bureau of Statistics mortality coding process \\
\hline $\begin{array}{l}\text { Medical cause of } \\
\text { death }\end{array}$ & As determined by the coroner \\
\hline $\begin{array}{l}\text { Incident date and } \\
\text { time }\end{array}$ & When the incident that caused death occurred \\
\hline $\begin{array}{l}\text { Mechanism and } \\
\text { object of injury }\end{array}$ & Mechanism by which death occurred/objects involved in the death \\
\hline Geocoding result & $\begin{array}{l}\text { Statistical areas } 3 \text { and } 4 \text { of residential address at the time of death. Statistical areas are provided by the } \\
\text { Australian Statistical Geography Standard for use by the Australian Bureau of Statistics and others to } \\
\text { analyse spatially integrated information }\end{array}$ \\
\hline
\end{tabular}

each contact, mental health diagnoses associated with contact and type of service.

\section{Data linkage}

The data linkage in this study will adhere to the separation principle for data linkage. Identifying information is supplied to the data linkage staff by the NCIS data custodians. Once the relevant linkage processes have been performed, deidentified data will then be available to researchers for analyses.

The separation principle will be maintained throughout the data linkage process as follows:

1. The researchers will not have access to identifying information once they have received the linked data.

2. Only approved research personnel will have access to the linked data and perform data analysis.

\section{Planned data analysis}

Aim 1: to describe health service utilisation rates (both general and mental health) in the year prior to death by suicide and how this varies by individual case characteristics.

Using descriptive statistics, we will comprehensively describe and characterise the healthcare utilisation in the cases within our sample in the year preceding suicide death by using tMBS data supplied by AIHW. Additionally, healthcare utilisation for the NSW cases will be described and categorised using the NSW Health data. Categorical data will be described using frequencies and percentages.

Generalised linear models will be used to determine predictors of healthcare contacts before death. The dependent healthcare utilisation variables we will investigate include ambulatory mental health contact, acute mental health contact and no mental health contact. Independent variables and covariates examined will include: age, sex, socioeconomic status (SES) and class of medicine prescribed. ORs with $95 \%$ CIs will be reported for each predictor.

Spatial maps of age and sex standardised suicide rate according to the presence or absence of healthcare within a month of death will be generated for Australia using statistical area 3 (SA3) regions of residence at the time of death.

Aim 2: to describe prescribed medicines use in the year prior to death by suicide, medicines used in suicide by poisoning and how this varies by individual case characteristics.

Using descriptive statistics, we will comprehensively describe and characterise the dispensed prescription medication use in our sample in the year preceding suicide death using the PBS data supplied by AIHW. Categorical data will be described using frequencies and percentages.

For the prescription medicines that are not used to treat mental illness, such as antibiotics, ACE inhibitors, gabapentinoids or isotretinoin, we will conduct disproportionality analysis ${ }^{18}$ to determine whether the rate of use in suicide cases is above that reported in the Australian population using other available sources of national representative PBS dispensing data.

Generalised linear models will be used to determine the predictors of prescribed medicine use patterns including: cases not dispensed a psychotropic medicine, cases non-compliant with psychotropic medicine at the time of death, initiation of psychotropic medicine within 1 month of death and discontinuation of psychotropic medicine within 1 month of death. Independent variables and covariates examined will include age, sex, SES, method of death and class of medicine prescribed. ORs with $95 \%$ CIs will be reported for each predictor.

Spatial maps of suicide according to the presence or absence of medicine within a month of death will be 
generated for Australia using SA3 regions of residence at the time of death.

\section{Patient and public involvement statement}

There were no funds or time allocated for the patient and public involvement, so we were unable to involve the public in the design of the study. However, we will develop a representative public committee to help us develop our dissemination strategy to mental health advocacy groups and the community.

\section{ETHICS AND DISSEMINATION}

This project involves the use of sensitive and confidential data. Data will be linked using a third-party privacypreserving protocol meaning that investigators will not have access to identifiable information once the data have been linked. Statistical analyses will be carried out in a secure environment. ASHLi has been approved by the following data custodians and ethics committees: (1) the National Coronial Information System (NCIS) (REF: M0400), (2) the Justice Department Human Research Ethics Committee (REF: CF/17/23250), (3) the Western Australian Coroners Court (REF: EC 14/18 M0400); (4) the Australian Institute of Health and Welfare (REF: EO2017/4/366), (5) the Department of Health and (6) NSW Population \& Health Services Research Ethics Committee (REF:2017/HRE1204). Findings will be published in peer-reviewed journals, presented at conferences and communicated to regulatory authorities, clinicians, mental health advocacy groups and policy-makers.

\section{DISCUSSION}

Australia is one of the few countries in the world with an investment in data linkage infrastructure to allow a project of this scope to be achieved. This study will significantly advance knowledge of health service and medicine utilisation prior to suicide in Australia. The project outcomes will have a significant impact on suicide prevention initiatives by identifying gaps in our national suicide prevention strategy, and subsequently providing information on ways to optimise future approaches.

ASHLi will result in the following central outcomes.

\section{Identifying the reach of suicide prevention initiatives delivered via health services}

By identifying population characteristics associated with different types of health service use, this analysis will provide important information for targeting suicide initiatives. For example, if one type of service is used more commonly in younger female individuals then reinforcing tailored programmes toward this group within that service may increase the efficacy of suicide prevention compared with broadly focused interventions. Furthermore, locating where high/low service use occurs and determining when, in relation to suicide, particular services are most commonly accessed, the analysis will provide specific information about where and when to reinforce or redirect our current strategies.

Highlighting barriers to health service utilisation

By interpreting health service use in concert with health service location we will shed light on whether barriers to health service utilisation may be due to intrinsic factors (unwillingness by the individual to seek help) versus extrinsic factors (remoteness/lack of services). The former will highlight groups of people for whom non-clinical forms of intervention may be the most efficacious and where they should be targeted (ie, online therapy, restricting means of suicide and public health campaigns).

\section{Providing information to help target means restriction}

Means restriction is the primary mode of suicide prevention applicable to those who do not access health services. Our analysis of the methods of suicide that are most commonly used in these people will provide information on priority suicide methods to target, in what populations and where.

\section{Highlighting potentially harmful medicine use}

Information on medicines used in overdose will have important implications for regulatory authorities and prescribers, especially when psychotropic medicine use occurs in the absence of mental health service utilisation, which is against best practice recommendations. We will also provide further evidence regarding concerns about the prosuicidal effects of several non-psychotropic medicines.

\section{Foundational research for future studies}

The rich data source created in order to achieve the aims of the current proposal will be used for many further analyses by the study team. For example, with the addition of appropriate data from the general population, we can zoom in on particular medicine classes of interest and conduct disproportionality analysis to assess how the use of medicines by our sample differs compared with the general population.

\section{Limitations}

There are a number of limitations associated with the nature of the data used in this study. First, there will be an under-ascertaininment of health service use for the cases in our study. MBS data do not include enough detail on mental health services provided in the hospital to allow the identification of hospitalised self-harm and other emergency psychiatric presentations. Health service data collected by State governments include detailed data on hospitalisations and non-hospital mental health services, therefore is not constrained by the same limitations. Therefore, we will supplement the MBS mental health data using NSW state data collections. Initially, we have chosen to use this approach in NSW given its large population (around one third of Australians). By comparing the estimates we receive from MBS alone to MBS plus NSW 
state collections, we will be able to estimate the degree of under-ascertainiment in other states in Australia and report on this accordingly.

Medicine use will also be limited by under-ascertainment. It is possible to obtain scripts outside the PBS, such as private prescriptions, medicines dispensed within public hospitals to inpatients or opioid maintenance treatments (methadone and buprenorphine) will not be captured. The study will also not capture medicines sold over the counter, complementary or alternative medicines.

Another limitation is that all cases in the study have died from suicide, therefore, the risk of suicide associated with any healthcare or medicine use patterns cannot be established. This study will instead highlight these patterns, so that further studies with appropriate control groups can be established.

\section{Author affiliations}

${ }^{1}$ Discipline of Pharmacology, Clinical Pharmacology and Toxicology Research Group, Faculty of Medicine and Health, University of Sydney, Sydney, New South Wales, Australia

${ }^{2}$ Department of Forensic Medicine, Victorian Institute of Forensic Medicine, Monash University, Clayton, Victoria, Australia

${ }^{3}$ Medicines Policy Research Unit, Centre for Big Data Research in Health, University of New South Wales, Sydney, New South Wales, Australia

${ }^{4}$ NSW Poisons Information Centre, The Children's Hospital at Westmead, Sydney, New South Wales, Australia

${ }^{5}$ Sydney Pharmacy School, University of Sydney, Sydney, New South Wales, Australia

${ }^{6}$ Calvary Mater Newcastle Hospital, University of Newcastle, Newcastle, New South Wales, Australia

${ }^{7}$ Translational Health Research Institute, Western Sydney University, Campbelltown, New South Wales, Australia

\section{Twitter Kate M Chitty @chitty_kate}

Acknowledgements The authors would like to thank the National Coronial Information System (NCIS), which is managed by the Victorian Department of Justice and Community Safety, Centre for Health Record Linkage (CHeReL) and the NSW Ministry of Health.

Contributors KC (principal investigator) and NB: conceptualisation of ASHLi. $\mathrm{KC}, \mathrm{JS}, \mathrm{RC}, \mathrm{AS}, \mathrm{NB}, \mathrm{AP}, \mathrm{GC}, \mathrm{JR}, \mathrm{S}-\mathrm{AP}$ : protocol design. KC and NJG: drafted the manuscript. All authors contributed to the final version.

Funding This study is funded by a grant from the National Health and Medical Research Centre (NHMRC, REF: APP1157757) and the Translational Australian Clinical Toxicology Research Group (TACT). An NHMRC Early Career Fellowship funds KC (Grant number: 1122362).

Disclaimer The funding organisations played no part in the design of the study nor in the preparation, review or approval of the manuscript.

Competing interests None declared.

Patient consent for publication Not required.

Provenance and peer review Not commissioned; peer reviewed for ethical and funding approval prior to submission.
Data availability statement No data are available. The data used in this protocol are highly sensitive and confidential. Only approved personnel may access the data. Researchers interested in collaborations or further information are invited to contact $\mathrm{KC}$ at kate.chitty@sydney.edu.au.

Open access This is an open access article distributed in accordance with the Creative Commons Attribution Non Commercial (CC BY-NC 4.0) license, which permits others to distribute, remix, adapt, build upon this work non-commercially, and license their derivative works on different terms, provided the original work is properly cited, appropriate credit is given, any changes made indicated, and the use is non-commercial. See: http://creativecommons.org/licenses/by-nc/4.0/.

\section{ORCID iDs}

Andrea Schaffer http://orcid.org/0000-0002-3701-4997

Rose Cairns http://orcid.org/0000-0002-8946-5079

Nicole J Gonzaga http://orcid.org/0000-0002-9596-4894

\section{REFERENCES}

1 Who: preventing suicide: a global imperative2014Geneva

2 Australian Bureau of Statistics. 3303.0 - Causes of Death, Australia, 2018.2019

3 Krysinska K, Batterham PJ, Tye M, et al. Best strategies for reducing the suicide rate in Australia. Aust N Z J Psychiatry 2016;50:115-8.

4 Zalsman G, Hawton K, Wasserman D, et al. Suicide prevention strategies revisited: 10-year systematic review. Lancet Psychiatry 2016;3:646-59.

5 Sinyor M, Howlett A, Cheung AH, et al. Substances used in completed suicide by overdose in Toronto: an observational study of coroner's data. Can J Psychiatry 2012;57:184-91.

6 Stene-Larsen K, Reneflot A. Contact with primary and mental health care prior to suicide: a systematic review of the literature from 2000 to 2017. Scand J Public Health 2019;47:9-17.

7 Ahmedani BK, Stewart C, Simon GE, et al. Racial/Ethnic differences in health care visits made before suicide attempt across the United States. Med Care 2015;53:430-5.

8 Sveticic J, Milner A, De Leo D. Contacts with mental health services before suicide: a comparison of Indigenous with non-Indigenous Australians. Gen Hosp Psychiatry 2012;34:185-91.

9 O'Neill S, Graham B, Ennis E. Prescribed pain and mental health medication prior to suicide: a population based case control study. $J$ Affect Disord 2019;246:195-200.

10 Ilgen MA, Kleinberg F, Ignacio RV, et al. Noncancer pain conditions and risk of suicide. JAMA Psychiatry 2013;70:692-7.

11 Ferrari AJ, Norman RE, Freedman G, et al. The burden attributable to mental and substance use disorders as risk factors for suicide: findings from the global burden of disease study 2010. PLoS One 2014;9:e91936.

12 Hawton K, Bergen H, Simkin S, et al. Toxicity of antidepressants: rates of suicide relative to prescribing and non-fatal overdose. $\mathrm{Br} \mathrm{J}$ Psychiatry 2010;196:354-8.

13 AlHW: Medicare-subsidised GP allied health and specialist health care across local areas: 2013-14 to 2017-18 2019.

14 AlHW. Australia's health 2016. Australia's health No. 15. cat. No. AUS 199. Canberra: AlHW, 2016.

15 AlHW. Mental health services in brief 2019.

16 Mellish L, Karanges EA, Litchfield MJ, et al. The Australian pharmaceutical benefits scheme data collection: a practical guide for researchers. BMC Res Notes 2015;8:634.

17 Australian Government Department of Health. Australian statistics on medicine 2011. Canberra: Australian Government Department of Health, 2013.

18 Caster O, Aoki Y, Gattepaille LM, et al. Disproportionality analysis for pharmacovigilance signal detection in small databases or subsets: recommendations for limiting false-positive associations. Drug Saf 2020;43:479-87. 